AIP Applied Physics

\title{
Time resolved characterization of a free-burning argon arc after ignition by optical emission spectroscopy
}

Shuiliang Ma, Hongming Gao, and Lin Wu

Citation: J. Appl. Phys. 110, 026102 (2011); doi: 10.1063/1.3610419

View online: http://dx.doi.org/10.1063/1.3610419

View Table of Contents: http://jap.aip.org/resource/1/JAPIAU/v110/i2

Published by the American Institute of Physics.

\section{Related Articles}

Implementation of moiré-schlieren deflectometry on a small scale fast capillary plasma discharge J. Appl. Phys. 111, 103301 (2012)

Simultaneous translational temperature measurements of different atomic species in plasma flows using scanning Fabry-Perot interferometry

Rev. Sci. Instrum. 83, 053111 (2012)

Quantitative measurement of hard x-ray spectra for high intensity laser produced plasma

Rev. Sci. Instrum. 83, 053502 (2012)

$\mathrm{X}$-ray conversion efficiency in vacuum hohlraum experiments at the National Ignition Facility

Phys. Plasmas 19, 053301 (2012)

Synergetic effects of double laser pulses for the formation of mild plasma in water: Toward non-gated underwater laser-induced breakdown spectroscopy

J. Chem. Phys. 136, 174201 (2012)

\section{Additional information on J. Appl. Phys.}

Journal Homepage: http://jap.aip.org/

Journal Information: http://jap.aip.org/about/about_the_journal

Top downloads: http://jap.aip.org/features/most_downloaded

Information for Authors: http://jap.aip.org/authors

\section{ADVERTISEMENT}

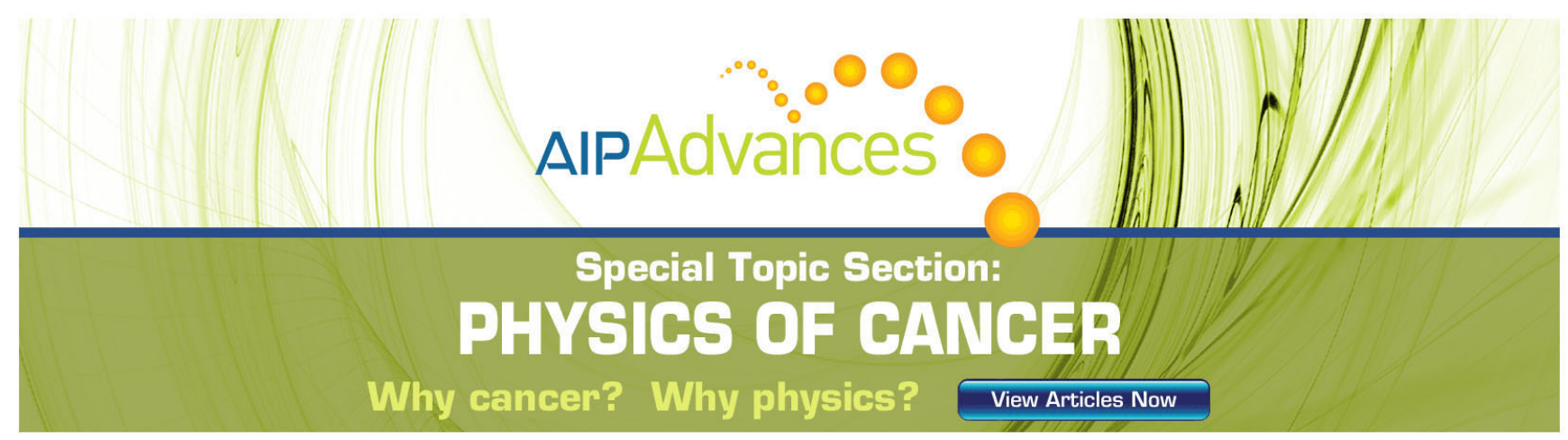




\title{
Time resolved characterization of a free-burning argon arc after ignition by optical emission spectroscopy
}

\author{
Shuiliang Ma, ${ }^{1,2, a)}$ Hongming Gao, ${ }^{1}$ and Lin $\mathrm{Wu}^{1}$ \\ ${ }^{1}$ State Key Laboratory of Advanced Welding Production Technology, Harbin Institute of Technology, Harbin \\ 150001, People's Republic of China \\ ${ }^{2}$ Plasma Research Laboratory, Australian National University, Canberra ACT 0200, Australia
}

(Received 8 April 2011; accepted 12 June 2011; published online 20 July 2011)

\begin{abstract}
Time resolved properties of a free-burning argon arc after ignition have been characterized using optical spectroscopic method. After ignition, when the arc current keeps constant, the plasma temperature decreases with time at any position of the arc. The decrease of the plasma temperature is associated with the increase of the arc cathode surface temperature. It is suggested that the variation of the cathode surface temperature, which changes the current density distribution over the cathode surface, leads to the decrease of the plasma temperature in the free-burning arc after ignition. (C) 2011 American Institute of Physics. [doi:10.1063/1.3610419]
\end{abstract}

Arc plasmas have been widely used in industrial areas, such as welding, cutting, spraying, and waste treatment. ${ }^{1,2}$ In order to control and improve these applications, a better understanding of the physical processes occurring in the plasma, especially in the cathode region, is important, which requires the plasma properties to be characterized.

Over the past few decades, a large number of studies have been devoted to such arc plasmas in both experiment ${ }^{3-11}$ and modeling. ${ }^{12-16}$ Using optical emission methods, arc properties, including the cathode surface temperature, ${ }^{3-8}$ the plasma temperature, ${ }^{5-9}$ and so on, have been determined and the effects of different arc conditions, e.g., the operation parameters $^{3,8,9}$ and the cathode shapes and materials, ${ }^{4-6}$ have been extensively investigated. On the other hand, the recent remarkable progress in arc theory enables the electrodes, the sheath regions, and the arc plasma to be included in arc models. ${ }^{14,15}$ Based on these models, the arc properties under different conditions have been predicted, which are in good agreement with experimental results (see Refs. 14 and 15 and references therein). Both experiments and modeling have shown the important role of the cathode energy transfer on arc properties. ${ }^{6,7,13,16}$ For example, the cathode cone angles effect the cathode surface temperature distribution and, consequently, effect the plasma temperature, ${ }^{6,13}$ and different arc cathode modes and the transitions between them are considered due to the bifurcation of energy balance problem in the cathode region. ${ }^{7,16}$

In spite of considerable research and development, the mechanisms in the arc cathode region are still not fully understood, due partly to the multiplicity and diversity of the processes involved and their interplay and partly to the extreme conditions in this region. In the case of high current arcs, most spectroscopic measurements are limited to steady state arcs, ${ }^{5-9}$ whereas there are few cases in which the transient properties in the arc cathode region have been investigated using spectroscopic methods. The time-dependent properties can provide more information about the processes

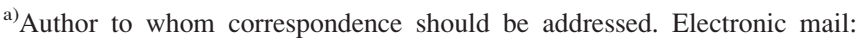
shlgma@126.com.
}

in the arc and are also important for modeling. Based on a filter spectrometer and a high-speed camera, we present observations of the temporal evolution of plasma temperatures in a free-burning arc in argon after ignition and interpret the mechanism behind this phenomenon.

The arc plasma was ignited by high-frequency pulses with a welding power supply (Fronius, MW3000) and free burning in argon $(99.9 \%$ pure, flow rate $10 \mathrm{~L} / \mathrm{min}$ ) at atmospheric pressure between two water-cooled electrodes. The cathode was a ceriated tungsten rod $\left(2 \% \mathrm{Ce}_{2} \mathrm{O}_{3}\right.$ by mass, diameter $3.2 \mathrm{~mm}$ ) ground to a conical tip with an included angle of $60^{\circ}$, and the anode was a copper plate situated vertically below the cathode with a distance of $5 \mathrm{~mm}$ from the cathode tip. The arc current was 200 A, which has a fluctuation less than $0.5 \%$.

Light emissions radiated from the arc were recorded with a filter spectrometer consisting of lenses and neutraldensity and interference filters together with a high-speed camera (Dalsa, CA-D6-0256W). The interference filters with the center wavelength at 794.8 and $780 \mathrm{~nm}$ and a full width at half maximum of about $3 \mathrm{~nm}$ have been used for the measurement of the Ar I $794.8 \mathrm{~nm}$ line intensity and the near continuum radiation, respectively. The camera captures 8 -bit gray images $(260 \times 260$ pixels corresponding to the CCD sensor size of $2.6 \times 2.6 \mathrm{~mm}^{2}$ ) with a time resolution of 955 frames/s. The spatial resolution in the measurement is about $0.09 \mathrm{~mm}$. As an example, Fig. 1 shows a few frames of the arc intensity images of the Ar I $794.8 \mathrm{~nm}$ line in the arc current increasing and constant stages. The arc was observed in a direction perpendicular to the $x-z$ plane. The origin of the coordinate system was set at the cathode tip, and the positive direction of the $z$-axis pointed from the cathode to the anode.

Temporal evolutions of the Ar I $794.8 \mathrm{~nm}$ line intensity at several axial positions in the arc with distances of 0.1 to $4 \mathrm{~mm}$ from the cathode tip are shown in Fig. 2. In the first 1 $\mathrm{s}$ time after the arc was ignited, the current of the arc increased in steps gradually, which is clearly reflected from the variation of the arc intensity. After $1 \mathrm{~s}$ time, the arc current reached the set value $200 \mathrm{~A}$ and was maintained constant. However, the arc intensities at different positions of 


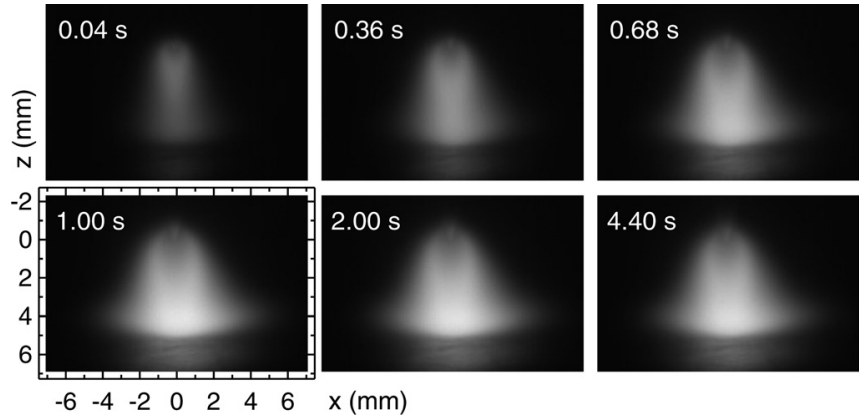

FIG. 1. A few frames of the Ar I $794.8 \mathrm{~nm}$ line intensity images acquired in a free-burning argon arc after ignition. The current of the arc gradually increased in steps to $200 \mathrm{~A}$ in $1 \mathrm{~s}$ time after the arc ignition and then was held constant.

the arc varied with time: in the cathode region $(z \leq 1 \mathrm{~mm})$, there is a minor increase in the intensity, and in regions far from the cathode $(z>2 \mathrm{~mm})$, the intensity has a gradual decreasing trend. Since the physical conditions were kept the same during the measurement, the variation of the intensity is most likely due to the change of the arc properties.

To reconstruct the radial plasma emission coefficients, side-on measured spectral line intensity profiles were symmetrized, smoothed, and Abel inverted. Based on the assumption of local thermodynamic equilibrium (LTE), the plasma temperatures then were derived from the local emission coefficients using a modified Fowler-Milne method. ${ }^{17}$ Figure 3(a) shows the temporal evolution of the plasma temperature at three axial positions of the arc. At the first $1 \mathrm{~s}$ time after the arc was ignited, the temperature increases rapidly due to the increase of the arc current. Then, the arc current was held constant, while the temperature decreased gradually. The temperature near the cathode has the largest decreasing trend: the decrease in the temperature is about $3000 \mathrm{~K}$ for $z=0.5 \mathrm{~mm}$ compared with $750 \mathrm{~K}$ for $z=2.5$ $\mathrm{mm}$. The axial and radial distributions of the plasma temperature at three time points also show that, at any position of the arc, the temperature decreases with time (see Figs. 3(b) and 3(c)). The departure of the plasma from LTE cannot explain the decrease in temperature because the regions far from the cathode are usually thought of in LTE. ${ }^{6}$ The exten-

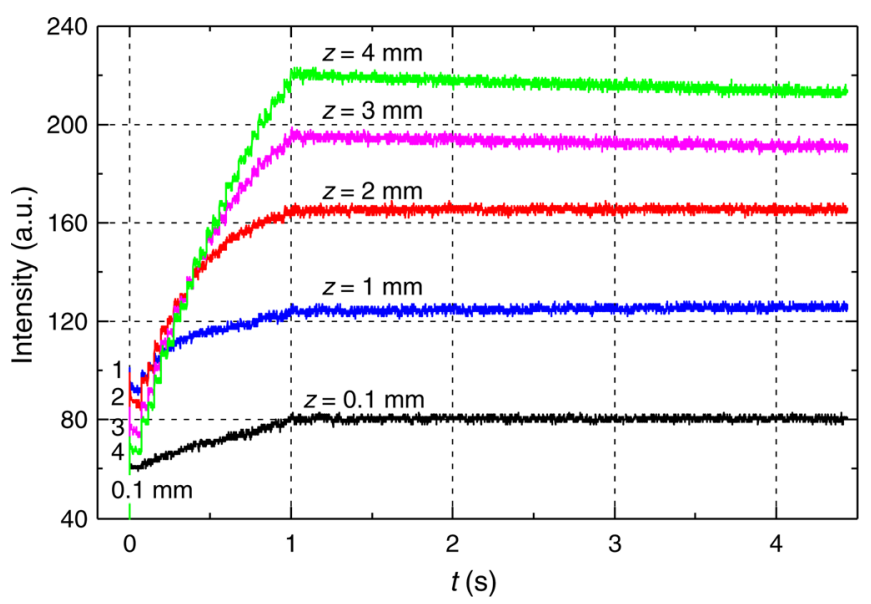

FIG. 2. (Color online) Temporal evolution of the Ar I $794.8 \mathrm{~nm}$ line intensity at several axial positions of the 200-A free-burning arc in argon. Other conditions are the same as Fig. 1.

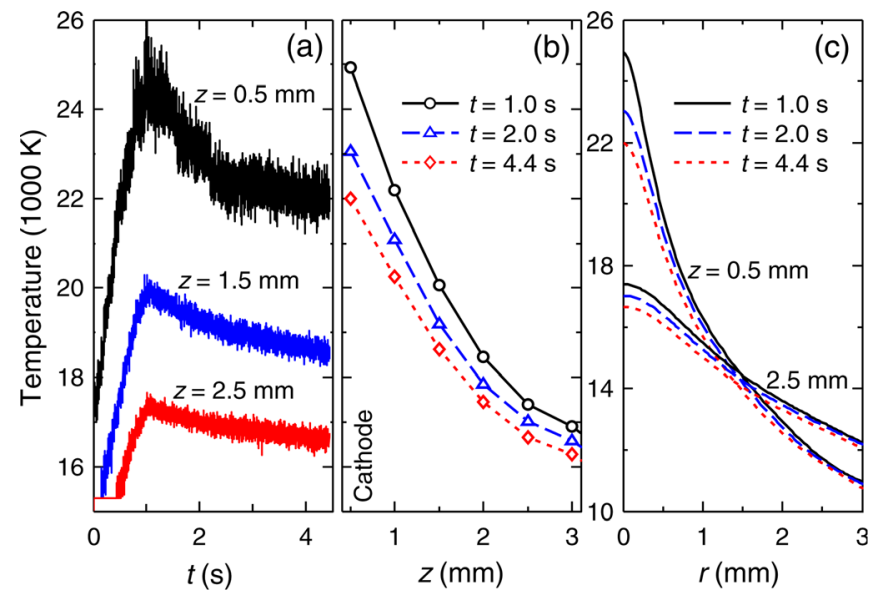

FIG. 3. (Color online) Temporal, axial, and radial distributions of the plasma temperature derived based on the measured intensity images for the 200-A free-burning arc in argon, as shown in Fig. 1.

sion of the cathode electrode due to heat expansion is also not responsible for the temperature decrease: if this is true, the temperature will increase rather than decrease with time, since the temperature in the cathode region has a much higher value. Considering all these factors and the variation of the measured intensity profiles in Fig. 2, it is believed that the plasma temperature profiles determined correctly reflect the change of the arc state.

It is difficult to determine the cathode surface condition from the measured Ar I $794.8 \mathrm{~nm}$ line intensities, due to the strong intensity of the arc spectral line and that reflected from the cathode. Therefore, the arc continuum radiations at $780 \mathrm{~nm}$ were recorded in another run with the same arc parameters. Figure 4 shows the continuum radiation profiles along the arc axis with lateral distances of $0,0.27$, and 0.82 $\mathrm{mm}$ from the axis center measured at three different time points. The profiles show that, for $z<-1.2 \mathrm{~mm}$, the intensity increases with time, while, for $z>-1.2 \mathrm{~mm}$, the intensities from the cathode and from the arc both decrease with time. The axial length of the arc-cathode interaction area was found to be about $1 \mathrm{~mm}$ (see also Ref. 3). Hence, the intensity at $z<-1.2 \mathrm{~mm}$ can be considered due completely to the thermal radiation from the cathode surface, which indicates that the cathode surface temperature was increasing after the arc ignition. In contrast, the intensity from the arc-cathode interaction area $(z>-1.2 \mathrm{~mm})$ consists of the radiations contributed from the cathode surface and from the arc as well as the reflection by the cathode. Previous spectroscopic measurements have shown that the radiation reflected from the cathode becomes negligibly low at the cathode edge, ${ }^{5}$ thus the measured intensity in this area is due mainly to radiations from the arc and from the cathode surface. To remove the radiation from the arc, a modified Abel inversion can be used, ${ }^{5}$ which, however, will introduce large uncertainties. Considering the relatively high spatial resolution in the measurement, the intensities from the arc at two near pixels of the image should be approximately the same. Hence, the radiation at the edge of the cathode contributed from the arc can be simply removed by subtracting half of the intensity at the near pixel, which views only the light from the arc. Figure 5 shows the temporal evolution of the intensities at 


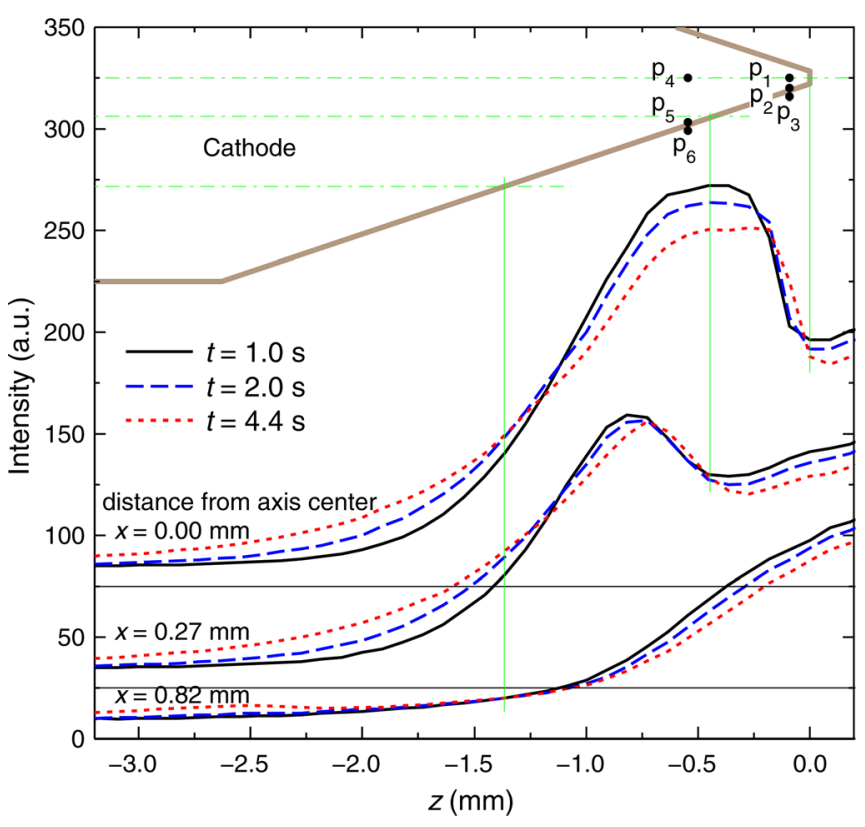

FIG. 4. (Color online) Axial profiles of the plasma continuum radiation at $780 \mathrm{~nm}$ measured at 1.0, 2.0, and $4.4 \mathrm{~s}$ after the arc ignition. The dash-dotted lines in the cathode area show the positions of the axial radiation distributions with distances of $0,0.27$, and $0.82 \mathrm{~mm}$ from the arc axis center. The intensity profiles at different lateral positions are offset from the nearest one for clarity. The positions of the six points $p_{1}$ to $p_{6}$ are also shown.

six positions of the arc cathode region, as marked by the black points in Fig. 4. Points $p_{1}$ and $p_{4}$ are located at the arc axis with distances of 0.09 and $0.55 \mathrm{~mm}$ from the cathode tip, respectively. Points $p_{2}$ and $p_{5}$ are very close to the cathode edge, and points $p_{3}$ and $p_{6}$ are the neighbor pixels of $p_{2}$ and $p_{5}$, respectively. The deduced intensity profiles at $p_{2}$ and $p_{5}$ due to the radiation from the cathode surface are also shown in Fig. 5, as labeled by $I_{2}-I_{3} / 2$ and $I_{5}-I_{6} / 2$. These profiles indicate that the cathode surface temperatures at $p_{2}$ and $p_{5}$ were increasing after the arc ignition. Therefore, based on the variation of the radiations from the arc cathode (Figs. 4 and 5) we can conclude that the cathode surface temperature at any position increases with time in the current constant stage after the arc was ignited.

The arc cathode surface temperature is determined by the heat balance of processes, such as ohmic heating, thermal conduction in cathode bulk, thermionic cooling, and heat transfer from the plasma to the cathode. ${ }^{6,13}$ After the arc was ignited, compared with the heat loss terms, the heat inputs, including ohmic heating and heat transfer from the plasma to the cathode, are the dominant factors, thus the cathode surface temperature increases with time. The increase in the cathode surface temperature enlarges the region that contributes to the arc current by thermionic emission. Therefore, the distribution of the current density over the cathode surface expands and the maximum current density decreases. Due to the more uniformly distributed current density, the plasma temperature consequently decreases. Other experimental measurements with different cathode parameters have also demonstrated the close relationship between the plasma temperature and the cathode surface temperature. ${ }^{6,7}$ In our case, the cathode sur-
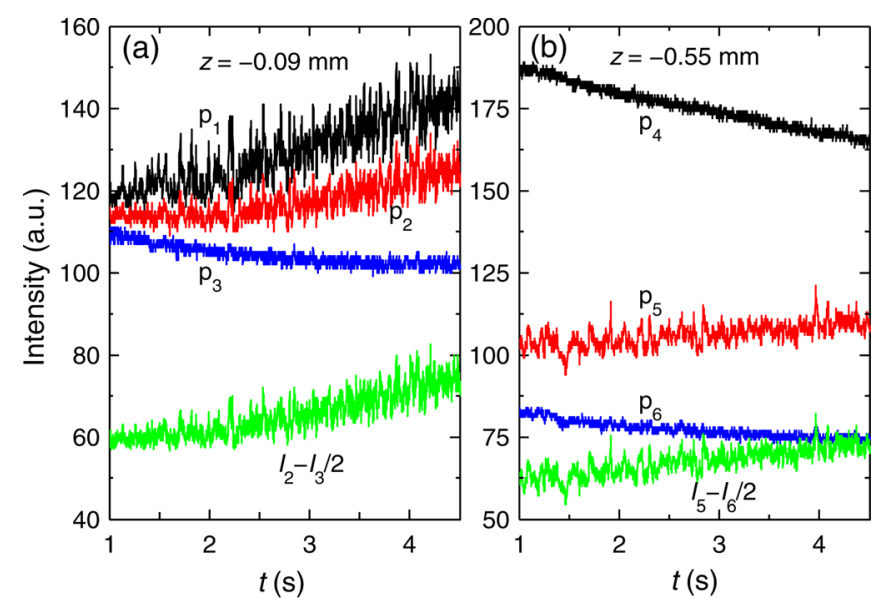

FIG. 5. (Color online) Temporal evolution of the plasma continuum radiation of the 200-A free-burning arc at several cathode positions, $p_{1}$ to $p_{6}$, as shown in Fig. 4, and of the radiation emitted from the cathode surface, $I_{2}-I_{3} / 2$ and $I_{5}-I_{6} / 2$, at points $p_{2}$ and $p_{5}$, respectively, with distances from the cathode tip of -0.09 and $-0.55 \mathrm{~mm}$.

face temperature determines the cathode current density distribution and thus affects the plasma temperature.

In conclusion, we showed that the measured plasma temperature in a free-burning argon arc was decreasing with time after the arc ignition, which is associated with the increase of the cathode surface temperature. This phenomenon indicated the important effect of the cathode surface temperature on the plasma temperature. For further investigations, it is necessary to determine both the cathode surface temperature and current density distributions. These measurements together with modeling results will help us for a better understanding of the mechanism in the arc cathode region.

${ }^{1}$ P. Fauchais and A. Vardelle, IEEE Trans. Plasma Sci. 25, 1258 (1997).

${ }^{2}$ P. Fauchais and A. Vardelle, Plasma Phys. Controlled Fusion 42, B365 (2000).

${ }^{3}$ J. A. Sillero, D. Ortega, E. Muñoz-Serrano, and E. Casado, J. Phys. D: Appl. Phys. 43, 185204 (2010).

${ }^{4}$ M. Ushio, A. A. Sadek, and F. Matsuda, Plasma Chem. Plasma Process. 11, 81 (1991).

${ }^{5}$ J. Haidar and A. J. D. Farmer, Rev. Sci. Instrum. 64, 542 (1993).

${ }^{6}$ J. Haidar and A. J. D. Farmer, J. Phys. D: Appl. Phys. 27, 555 (1994).

${ }^{7}$ N. K. Mitrofanov and S. M. Shkol'nik, Tech. Phys. 52, 711 (2007).

${ }^{8}$ X. Zhou and J. Heberlein, Plasma Chem. Plasma Process. 16, 229s (1996).

${ }^{9}$ G. N. Haddad and A. J. D. Farmer, J. Phys. D: Appl. Phys. 17, 1189 (1984).

${ }^{10}$ M. E. Rouffet, Y. Cressault, A. Gleizes, and J. Hlina, J. Phys. D: Appl. Phys. 41, 125204 (2008).

${ }^{11}$ B. Pokrzywka, S. Pellerin, K. Musiol, F. Richard, and J. Chapelle, J. Phys. D: Appl. Phys. 29, 2841 (1996).

${ }^{12}$ A. Gleizes, J. J. Gonzalez, and P. Freton, J. Phys. D: Appl. Phys. 38, R153 (2005).

${ }^{13}$ L. E. Cram, J. Phys. D: Appl. Phys. 16, 1643 (1983).

${ }^{14}$ A. B. Murphy, M. Tanaka, S. Tashiro, T. Sato, and J. J. Lowke, J. Phys. D: Appl. Phys. 42, 115205 (2009).

${ }^{15}$ M. Tanaka, K. Yamamoto, S. Tashiro, K. Nakata, E. Yamamoto, K. Yamazaki, K. Suzuki, A. B. Murphy, and J. J. Lowke, J. Phys. D: Appl. Phys. 43, 434009 (2010).

${ }^{16}$ M. S. Benilov, J. Phys. D: Appl. Phys. 41, 144001 (2008).

${ }^{17}$ S. Ma, H. Gao, and L. Wu, Rev. Sci. Instrum. 82, 013104 (2011). 